“ (C) 2018 IEEE. Personal use of this material is permitted. Permission from IEEE must be obtained for all other uses, in any current or future media, including

reprinting/republishing this material for advertising or promotional purposes, creating new collective works, for resale or redistribution to servers or lists, or reuse of any copyrighted component of this work in other works." 


\section{The Design of a Compact, Wide Bandwidth, Non- Foster-Based Substrate Integrated Waveguide Filter}

\author{
Ting Shi, Ming-Chun Tang \\ College of Communication Engineering, Chongqing \\ University, Chongqing, 400044, China \\ E-mail: tangmingchun@cqu.edu.cn
}

\author{
Richard W. Ziolkowski ${ }^{1,2}$ \\ ${ }^{1}$ University of Technology Sydney, Global Big Data \\ Technologies Centre, Ultimo NSW 2007, Australia, and the \\ ${ }^{2}$ Department of Electrical and Computer Engineering, \\ University of Arizona, Tucson, AZ 85721, USA \\ E-mail: Richard. Ziolkowski@uts.edu.au
}

\begin{abstract}
A compact, wideband, half-mode substrate integrated waveguide (HM-SIW) filter with internal non-Foster element is demonstrated. First, its passive version is simulated and measured. Next, by integrating an ideal tunable capacitor at the end of the central stub of the HM-SIW resonator, the frequency-agile characteristic of the tunable HM-SIW filter is investigated. Finally, a negative impedance converter (NIC) is developed to replace this tunable capacitor to design a new nonFoster filter. The non-Foster-based HM-SIW filter was realized. Its measured results indicate that it has an operational fractional bandwidth of $10.8 \%$ and an electrical size $0.118 \times 0.292 \lambda_{g}^{2}$, which is a 3.93 times bandwidth increase and a $\sim 12 \%$ electrical size reduction compared to its passive, fixed capacitance version.
\end{abstract}

Keywords-Half-mode substrate integrated waveguide filter, negative impedance converter, non-Foster element, wide bandwidth

\section{INTRODUCTION}

Substrate integrated waveguide (SIW) technology, due to its inherent advantages of high-Q factor, low loss, easy fabrication, high power capacity, etc, has been widely investigated for engineering applications into the circuit equipments/elements in microwave and millimeter-wave ranges [1]. Most recently, compact-sized SIW filters have attracted much attention. Typically, they have narrow operational bandwidths. Many methods have been reported to enhance their operational bandwidths, such as cascading multi-order resonators, loading periodical elements onto the main resonators, combining several adjacent modes, etc. While effective, these solutions inescapably require large footprints.

Non-Foster elements, which yield a negative slope of the reactance versus frequency, are traditionally conceived as circuits that incorporate negative capacitors and negative inductors [2]. Owing to their unique reactance characteristics over the passive elements, non-Foster elements have been successfully applied into many microwave systems to improve their operational performance characteristics. Examples include antennas, power amplifiers, absorbers, and metamaterials, to name a few.

In this paper, a compact, wideband, half-mode SIW (HMSIW) filter with internal non-Foster element will be demonstrated. The non-Foster element will be shown to increase the operational bandwidth of the HM-SIW filter while decreasing its electrical size.

\section{Compact, Passive, Tunable HM-SIW Filter Design}

Compared with standard SIW resonators, HM-SIW designs maintain their performance characteristics, but with only half the electrical size [3-4]. A basic compact passive rectangular HM-SIW filter is designed and shown in Fig. 1(a). It consists of an one-order HM-SIW resonator and two directly-connected $50 \Omega$ microstrip feed lines. As shown in Fig. 1 (b), its simulated (measured) $-10 \mathrm{~dB}$ impedance bandwidth is from $695-738 \mathrm{MHz}$ (696 to $734 \mathrm{MHz}$ ) with FBW is $\sim 6 \%$ ( $5.3 \%)$. The fabricated filter has a size of $0.186 \times 0.256 \lambda_{g}^{2}(48$ $\left.\times 66 \mathrm{~mm}^{2}\right)$. Furthermore, it is noted that its out-of-band suppression is poor.

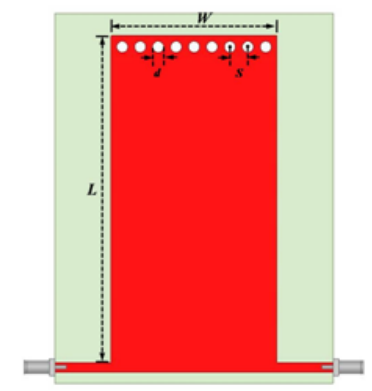

(a)

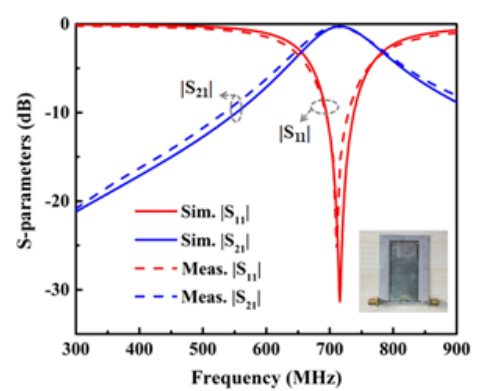

(b)
Fig. 1 Passive HM-SIW filter. (a) Geometry with $W=29.6 \mathrm{~mm}, L=56.3 \mathrm{~mm}$, $d=2.0 \mathrm{~mm}$ and $s=3.2 \mathrm{~mm}$. (b) Simulated and measured S-parameters.

By integrating an ideal tunable capacitor $C_{-} t$ (internal resistance $\mathrm{R}=0 \Omega$ ) into the open end of the HM-SIW resonator, a tunable HM-SIW filter is achieved. It is shown in Fig. 2(a). Sweeping the capacitance $C_{-} t$, the frequency-agile performance of this HM-SIW filter was studied and is shown in Fig. 2(b). As $C_{-} t$ was varied from 20 to $60 \mathrm{pF}$ in steps of $5 \mathrm{pF}$, the tunable $\mathrm{HM}$-SIW filter demonstrated excellent frequency-agile performance. It had a very good impedance match level $\left(\left|\mathrm{S}_{11}\right|_{\min }<-20 \mathrm{~dB}\right)$ with an average $\mathrm{FBW} \sim 3.6 \%$ at every discrete frequency state in the set. Additionally, these results indicate that the presence of this capacitor can be used to lower the resonance frequency significantly. 


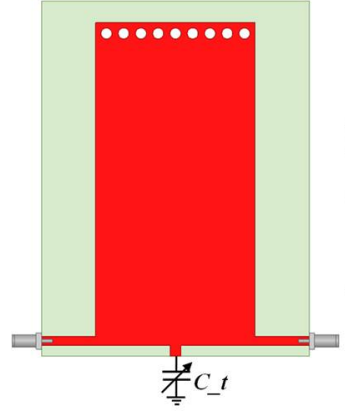

(a)

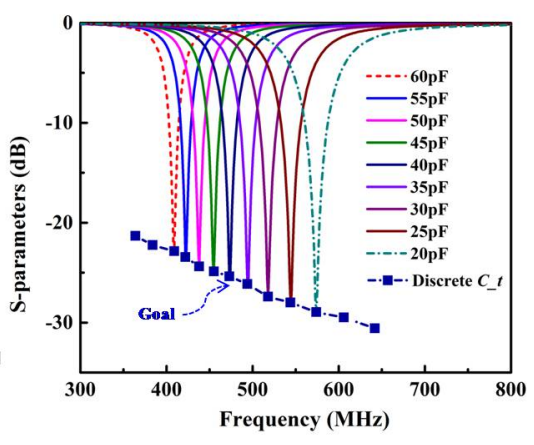

(b)
Fig. 2 Passive tunable HM-SIW filter. (a) Geometry. (b) Frequency-agile characteristics.

\section{AN IDEAL NON-FOSTER HM-SIW FILTER}

In order to study the bandwidth enhancement that a nonFoster version of the tunable capacitor could provide, the discrete ideal reactances associated with the tunable HM-SIW filter as $C_{-} t$ was varied were explored in a wider frequency range. As shown in Fig. 3, three lines, labeled Line_1, Line_2 and Line 3, respectively, are curve-fit to the discrete resonance frequencies produced by different $C_{-} t$ values over this frequency range. In detail, Line_1 was obtained with $C \_t$ varying from 150 to $20 \mathrm{pF}$. Line 2 and Line 3 were obtained for $C_{-} t$ values varying from 160 to $70 \mathrm{pF}$ and from 60 to 25 $\mathrm{pF}$, respectively. Clearly, these three lines fit different frequency ranges.

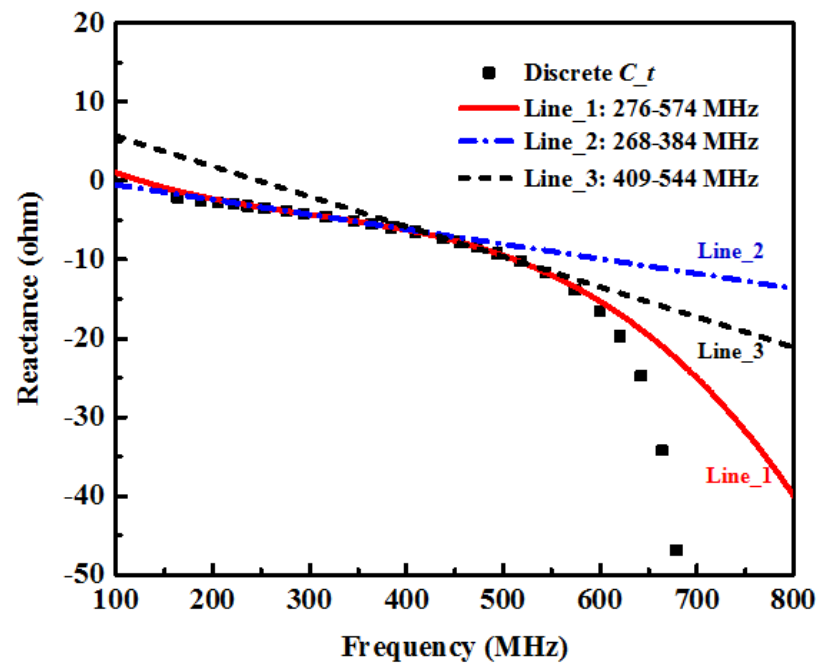

Fig. 3 Different curve fitting lines matched to the simulated reactance versus resonance frequency values.

As is shown in Fig. 4, three ideal negative impedance converter (NIC) circuits (their internal resistance, $0 \Omega$, and reactance operating as Line_1, Line_2 and Line_3, respectively) yield the HM-SIW filters that provide obvious bandwidth improvements. In detail, the ideal NIC circuit with its reactance values defined by Line_1 produces an HM-SIW filter with an instantaneous passband from 254 to $623 \mathrm{MHz}$ $(\mathrm{FBW}=84.1 \%)$. Similarly, the ideal NIC defined by Line_2 yields the non-Foster filter from 265 to $417 \mathrm{MHz} \mathrm{FBW}^{-}=$ $44.6 \%$ ). The ideal NIC defined by Line 3 provides the operation frequency band from 387 to $54 \overline{1} \mathrm{MHz}(\mathrm{FBW}=$ $33.4 \%$ ). As a reference, the HM-SIW filter would resonate at $400 \mathrm{MHz}$ with $\mathrm{FBW}=2.67 \%$ with a fixed $63 \mathrm{pF}$ capacitance. Briefly, the reactances provided by Line_1, Line_2, and Line 3 enable a bandwidth improvement as high as $29.6,16.9$ and 12.5 times, respectively.

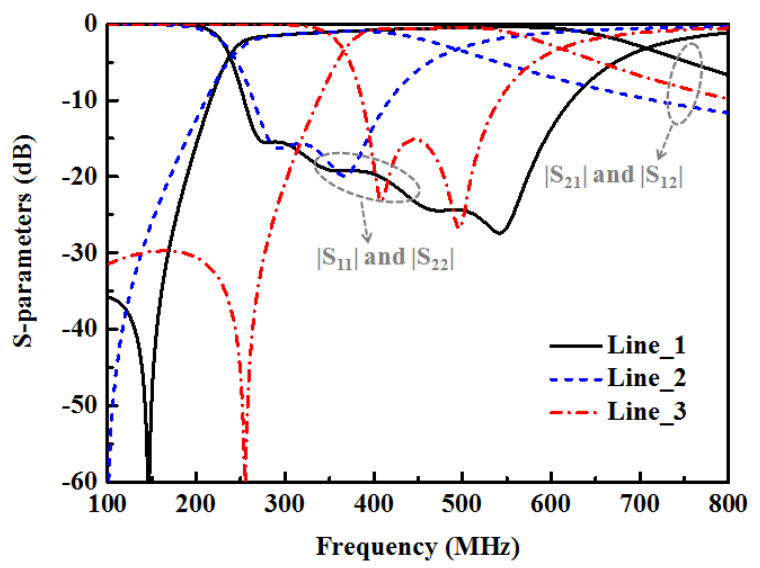

Fig. 4 Simulated S-parameters associated with the ideal non-Foster HM-SIW filters defined by the different reactance curve-fits

\section{NON-FOSTER HM-SIW FILTER}

A grounded NIC circuit based on cross-connecting two transistors, which was introduced originally by Linvill [23], is designed and revised, as shown in Fig. 5(a). Its fabricated layout and measurement setup are shown in Fig. 5(b). The measured Z-parameters of the experimentally-optimized NIC circuit are shown in Fig. 6. It is demonstrated that the resistance of the NIC circuit ranges from 0 to $10 \Omega$ in the frequency range from 381 to $451 \mathrm{MHz}$. This result was deemed to be low enough since the reactance values were very close to those specified by Line_3 in Fig. 3 .

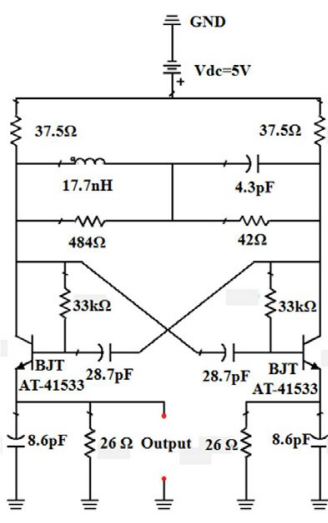

(a)

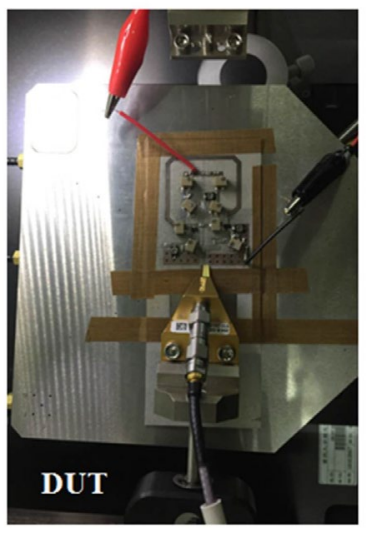

(b)
Fig. 5 Realized grounded NIC circuit design. (a) Circuit schematic. (b) Measurement setup. 
Integrating the experimentally-optimized NIC circuit with the HM-SIW filter, the final non-Foster HM-SIW filter was fabricated and measured. This prototype is shown in Fig. 7. The measured results demonstrate that the $-10 \mathrm{~dB}$ impedance bandwidth is from 401 to $447 \mathrm{MHz}(\mathrm{FBW}=10.8 \%)$, and the maximum insertion loss is $\sim 3.2 \mathrm{~dB}$. In the very wide frequency range from 360 to $500 \mathrm{MHz}$, the S-parameters maintain good complementarity. The $\left|S_{11}\right|$ and $\left|S_{22}\right|$ values were both slightly larger than $0 \mathrm{~dB}$ at the two edges of the passband. This phenomenon is readily attributed to some minor oscillations arising in the NIC circuit caused by the fabrication tolerances and random errors between the nominal and actual values of the components. In comparison to the passive, fixed capacitance reference version, the measured non-Foster HM-SIW filter operates in the range of 401-447 $\mathrm{MHz}$ with $\mathrm{FBW}=10.8 \%$, yields a $\sim 3.93$-fold bandwidth increase. Moreover, its stop-band rejection level has been improved significantly.

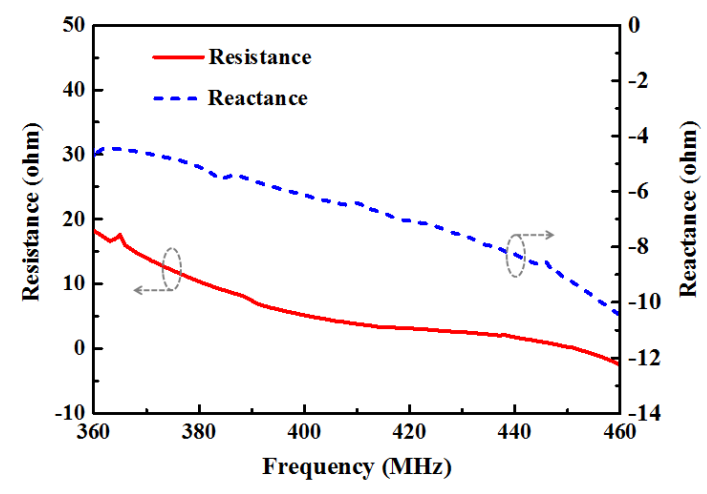

Fig. 6 Measured input impedance of the NIC circuit alone.

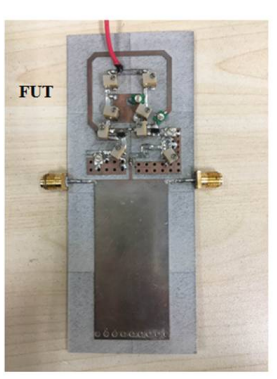

(a)

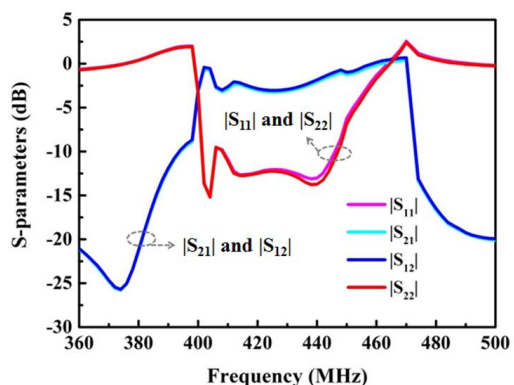

(b)
Fig. 7 Non-Foster HM-SIW filter. (a) Photo. (b) Measured S-parameters.

TABLE I. COMPARISONS BETWEEN THE NON-FOSTER FILTER AND THE COMPACT-SIZED SIW FILTERS REPORTED IN THE LITERATURE

\begin{tabular}{|c|c|c|c|}
\hline Refs. & Techniques & FBW $(\%)$ & $\begin{array}{c}\text { Electrical size } \\
\left(\lambda_{\mathrm{g}}{ }^{2}\right)\end{array}$ \\
\hline$[1]$ & 4-order SIW & 12.1 & $0.86 \times 0.86$ \\
\hline$[3]$ & 2-order HM-SIW & 10.8 & $0.45 \times 0.67$ \\
\hline$[4]$ & 3-order HM-SIW & 7.6 & $0.51 \times 0.21$ \\
\hline$[6]$ & 2-order QM-SIW & 5.9 & $\sim 0.158 \times 0.287$ \\
\hline$[7]$ & 4-order EM-SIW & 15.2 & $0.79 \times 0.36$ \\
\hline $\begin{array}{c}\text { This } \\
\text { work }\end{array}$ & non-Foster HM-SIW & 10.8 & $0.118 \times 0.292$ \\
\hline
\end{tabular}

In order to illustrate the advantages of our non-Foster technology in bandwidth improvement, Table I is provided in order to compare the bandwidth of our design with other reported compact-sized SIW/HM-SIW/QM-SIW [6] / EMSIW [7] filters. Table I demonstrates that it produces a much wider operational bandwidth than any of the much larger-sized examples.

\section{CONCLUSION}

A compact-sized, wide bandwidth, HM-SIW filter augmented with a non-Foster element was presented. A frequency-agile (tunable capacitor) HM-SIW filter was first designed and analyzed. Its operational mechanisms were characterized and verified with a measured prototype. A NIC circuit was designed, tested, and integrated into the HM-SIW filter. The measured results of this non-Foster HM-SIW filter demonstrated that it has a wide operational FBW $=10.8 \%$, and an electrically small size: $0.118 \times 0.292 \lambda_{g}{ }^{2}$. The application of this filter to achieve an enhanced bandwidth filtenna [8] will be discussed in our presentation.

\section{ACKNOWLEDGMENTS}

This work was supported in part by the National Natural Science Foundation of China contract number 61471072, in part by the Funding of the Innovative Leading Talents in Science and Technology of Chongqing contract number CSTCCXLJRC201705, in part by Funding of the Young Backbone Teachers in Colleges and Universities of Chongqing contract number 0307001104102, and in part by the Australian Research Council grant number DP160102219.

\section{REFERENCES}

[1] C. J. You, Z. N. Chen, X. W. Zhu, and K. Gong, "Single-layered SIW post-loaded electric coupling-enhanced structure and its filter applications," IEEE Trans. Microw. Theory Tech., vol. 61, no. 1, pp: 125-130, Jan. 2013.

[2] S. D. Stearns, "Stable band-pass non-Foster circuits," in Proc. 2015 IEEE International Symposium on Antennas and Propagation USNC/URSI National Radio Science Meeting, pp. 1386-1387, Jul. 2015.

[3] F. Chen, K. Song, B. Hu, and Y. Fan, "Compact dual-band bandpass filter using HM-SIW resonator and slot perturbation," IEEE Microw. Wireless. Compon. Lett., vol. 24, no. 10, pp. 686-688, Oct. 2014.

[4] Q.-L. Zhang, B.-Z. Wang, D.-S. Zhao, and K. Wu, "A compact halfmode substrate integrated waveguide bandpass filter with wide out-ofband rejection," IEEE Microw. Wireless. Compon. Lett., vol. 26, no. 7, pp. 501-503, Jul. 2016.

[5] J. G. Linvill. "Transistor negative-impedance converters," in Proc. IRE, vol. 40, pp. 725-729, Jun. 1953.

[6] A. P. Saghati, and K. Entesar, "Ultra-miniature SIW cavity resonators and filters," IEEE Trans. Microw. Theory Techn., vol. 63, no. 12, pp. 4329-4340, Dec. 2015.

[7] P. Li, H. Chu, and R.-S. Chen, "Design of compact bandpass filters using quarter-mode and eighth-mode SIW cavities,"IEEE Trans. Microw. Theory Tech., vol. 7, no. 6, pp. 956-963, Jun. 2017.

[8] M.-C. Tang, T. Shi, D. Li, Z. Wu, Y. Wang, Z. Yang, D. Li, M. Li, and R. W. Ziolkowski, "Compact, half-mode, substrate integrated cavitybacked slot antenna augmented with an internal non-Foster element to enlarge its instantaneous bandwidth," submitted to IEEE Trans. Antennas Propag., Feb. 2018. 